\title{
ORIGINAL RESEARCH \\ Subtraction 3D CT Angiography with the Orbital Synchronized Helical Scan Technique for the Evaluation of Postoperative Cerebral Aneurysms Treated with Cobalt-Alloy Clips
}

Y. Watanabe

N. Kashiwagi

N. Yamada

M. Higashi

T. Fukuda

S. Morikawa

Y. Onishi

K. lihara

S. Miyamoto

H. Naito

BACKGROUND AND PURPOSE: CT angiography (CTA) has been used for the evaluation of intracranial aneurysms and recently has been applied to assess postoperative aneurysms treated with titanium-alloy clips. We investigated the clinical usefulness of subtraction CTA by using the orbital synchronized helical scan technique (OSHST) for evaluating intracranial aneurysms surgically treated with cobalt-alloy clips.

MATERIALS AND METHODS: We scanned an agar gel phantom with a cobalt-alloy clip mounted in the center by using subtraction CT with and without OSHST. Eighteen patients (20 aneurysms) who underwent surgery with cobalt-alloy clips were postoperatively evaluated with subtraction CTA with OSHST, and the results were compared with those from digital subtraction angiography. Two neuroradiologists independently evaluated the 3D CTA images and source images with and without subtraction for the presence of residual flow in the aneurysm and stenotic change in parent or neighboring arteries.

RESULTS: For the phantom study, significantly fewer artifacts from clips were noted on images obtained by using subtraction CT with OSHST than on those obtained without OSHST. For the clinical study, subtraction CTA with OSHST also showed fewer clip artifacts than did conventional CTA. Image quality was poor, and we were unable to diagnose residual neck for $5 \%(1 / 20)$ with subtraction CTA with OSHST and 75\% (15/20) with conventional CTA. For evaluation of adjacent vessels, image quality was poor for none $(0 / 20)$ with subtraction CTA with OSHST and for 55\% (11/20) with conventional CTA. For subtraction CTA with OSHST, sensitivity in detecting residual neck was 1.0 , and specificity was 0.94. For conventional CTA, sensitivity and specificity were both 0.25 .

coNCLUSIONS: OSHST is a useful technique for subtracting cobalt-alloy clips, and subtraction CTA with OSHST is available for evaluating aneurysms after clipping with cobalt-alloy clips.

$\mathbf{S}^{\mathrm{s}}$ ubtraction CT angiography (CTA), which eliminates bone structures by using a precontrast scan, has become a useful and noninvasive technique to evaluate intracranial aneurysms, especially those adjacent to bone. ${ }^{1-3}$ The orbital synchronized helical scan technique (OSHST) was developed to permit 2 consecutive acquisitions along the same helical path, thereby reducing the misregistration between the 2 scans to enable acquisition of accurate subtraction images. ${ }^{4}$

CTA of the brain has recently been applied to assess aneurysms surgically occluded by the use of titanium-alloy clips. ${ }^{5-7}$ However, the strong artifacts from cobalt-alloy clips complicated assessment of the aneurysm neck and adjacent vessels ${ }^{8,9}$; it was thought that postoperative evaluation by using CTA was difficult for patients treated with cobalt-alloy clips.

To elucidate the clinical usefulness of subtraction CTA with OSHST for evaluating intracranial aneurysms surgically treated with cobalt-alloy clips, we examined the difference in subtraction images with or without OSHST in a phantom and compared findings from subtraction CTA with OSHST, conventional CTA, and digital subtraction angiography (DSA) in patients who had undergone cobalt-clip occlusion.

Received September 3, 2007; accepted after revision January 15, 2008.

From the Departments of Radiology (Y.W., N.Y., M.H., T.F., S. Morikawa, Y.O., H.N.) and Neurosurgery (K.I., S. Miyamoto), National Cardiovascular Center, Osaka, Japan; and Department of Radiology (N.K.), Osaka Medical Center for Cancer and Cardiovascular Disease, Osaka, Japan.

Please address correspondence to Yoshiyuki Watanabe, MD, PhD, 5-7-1 Fujishirodai, Suita Osaka, Japan 565-8565; e-mail: ywatanab@hsp.ncvc.go.jp

DOI 10.3174/ajnr.A1040

\section{Methods}

\section{Phantom Study}

We mounted a cobalt-alloy clip (Sugita Aneurysm Clip; Mizuho, Tokyo, Japan) in the center of an agar gel phantom and examined 2 consecutive helical CT scans to obtain the subtraction CT with a 16section CT scanner (Aquilion; Toshiba Medical Systems, Tokyo, Japan) by using following parameters: collimation, $0.5 \mathrm{~mm}$; helical pitch, 11/16; tube voltage, $120 \mathrm{kV}$; tube current, $200 \mathrm{mAs}$; rotation time, 0.6 seconds; reconstruction section width, $0.5 \mathrm{~mm}$; and reconstruction interval, $0.5 \mathrm{~mm}$. Two consecutive sets of helical scan data, with OSHST or without OSHST, were acquired separately, and each procedure was repeated 5 times. We subtracted the first scan from the second scan and recorded the x-ray tube start angle of 2 consecutive helical scans. The circled region of interest (40-mm diameter), which included the clip, was placed on the subtraction image, and the mean $\mathrm{CT}$ value and $\mathrm{SD}$ were measured.

\section{Patient Population}

We retrospectively reviewed 560 consecutive brain CTA examinations between January 2006 and March 2007. Eighty-six patients were examined for postoperative evaluation after placement of cobalt-alloy clips by using subtraction CTA with OSHST. We selected 18 patients (32-68 years of age; mean, 55.4 years; 8 men, 10 women) with a total of 20 aneurysms who underwent DSA within 6 months after or before CTA. Locations of the aneurysms were the following: internal carotid artery ( 9 cases), middle cerebral artery ( 7 cases), and anterior cerebral artery ( 4 cases). Seven patients had 1 clip, 8 patients had 2, 4 had 3, and 1 had 4 . 
This study proceeded in accordance with the ethics guidelines of our hospital, and each patient provided informed consent on admission.

\section{Imaging Study}

All CTA studies were performed by using a 16-section CT scanner with the following parameters: collimation, $0.5 \mathrm{~mm}$; helical pitch, 11/16; tube voltage, $120 \mathrm{kV}$; tube current, $300 \mathrm{~mA}$; rotation time, 0.6 seconds; reconstruction section width, $0.5 \mathrm{~mm}$; and reconstruction interval, $0.3 \mathrm{~mm}$. Pre- and postcontrast-enhanced scanning was performed with OSHST, and 2 consecutive scans were obtained under the same conditions. Contrast medium ( $350 \mathrm{mg} \mathrm{I} / \mathrm{mL}, 1.0 \mathrm{~mL} / \mathrm{kg}, 2.0$ $\mathrm{mL} / \mathrm{s}$ ) was injected via an antecubital vein, followed immediately by a saline flush. The scanning delay was set to 30 seconds. We used a specialized brain holder to minimize head movement during the helical scanning. Subtraction CT was calculated to subtract precontrast CT images from postcontrast CT images. Subtraction images were checked by a radiologist, and if they contained misregistration artifacts caused by movement of the patient's head, postcontrast images were corrected by a physician by using Volume Position Matching (Toshiba Medical Systems) (a manual method for correcting positional shift by adjusting image positions in 3D, resembling the "pixelshift" in DSA). The acquired subtraction and conventional (nonsubtraction) CTA images were transferred to a $3 \mathrm{D}$ workstation (Zio M900; Ziosoft, Tokyo, Japan) to generate 3D images by using volume rendering (VR). In subtraction CTA, the clips themselves were subtracted and not displayed on VR images. Therefore, the clips were extracted from the presubtraction images, and clip images were superimposed onto subtraction VR images if reviewers wanted to observe the relationship between vessels and clips.

DSA was performed by using a biplane DSA unit with rotational 3D DSA.

\section{Review Process}

Two neuroradiologists independently reviewed the DSA and CTA on workstations, with final determination reached by consensus. CTA images were reviewed independently of subtraction CTA and conventional CTA by using a VR image with reference to the source images and multiplanar reconstruction images. Both CTA images were evaluated for the presence of residual flow in the aneurysm and stenotic change in the parent or neighboring artery. If image quality was poor for estimation due to large artifacts from cobalt-alloy clips or inappropriate subtraction, it was diagnosed "not applicable."

\section{Statistical Analysis}

With an unpaired $t$ test, the region-of-interest data (mean and SD) obtained with subtraction images with OSHST were compared with those obtained with subtraction images without OSHST. The difference in X-ray tube start angle between 2 consecutive scans with OSHST was compared with that without OSHST. The McNemar test for paired proportions was used to determine if subtraction CTA with OSHST had more assessable image quality than that without OSHST. All tests were implemented by using the Statistical Package for the Social Sciences software (SPSS, Chicago, Ill). Statistical significance was established at $P=.05$.

\section{Results}

In the phantom, the subtraction image without OSHST showed the radial artifact, but with OSHST, few artifacts were apparent (Fig 1). Table 1 summarizes the results. The SD of the region of interest was significantly small in the subtraction image with OSHST, and the difference of $\mathrm{x}$-ray tube start angle with OSHST was nearly zero and significantly narrow compared with that without OSHST.

Despite using a head-immobilization device, Volume Position Matching was performed for 7 of 18 patients (39\%). The simple subtraction of precontrast and postcontrast datasets required $<1$ minute, and if Volume Position Matching was applied, then another 5-10 minutes was required for further analysis.

Table 2 summarizes CTA and DSA findings in the clinical study. The subtraction CTA with OSHST had fewer clip artifacts than did conventional CTA (Figs 2 and 3). Image quality was poor, and we were unable to diagnose the residual neck for only $5 \%(1 / 20)$ with subtraction CTA with OSHST and 75\% $(15 / 20)$ with conventional CTA. For evaluation of adjacent vessels, image quality was poor for none $(0 / 20)$ with subtraction CTA with OSHST and for 55\% (11/20) with conventional CTA. There were significant differences between the 2 methods for evaluation of both residual neck and adjacent vessels $(P<.01)$.

DSA depicted 4 residual necks or recurrent aneurysm in 20 postoperative aneurysms. Subtraction CTA with OSHST could reveal all 4 neck abnormalities, though conventional CTA depicted only 1 residual neck (Fig 2). For subtraction CTA, the sensitivity for detecting residual or recurrent aneurysm was 1.0 , and specificity was 0.94 . For conventional CTA, sensitivity and specificity were both 0.25 .

For subtraction CTA, the sensitivity for detecting parent or neighboring artery stenosis was 1.0 , and specificity was 0.95 . For conventional CTA, sensitivity was 0 , and specificity was 0.32. Pseudostenosis of a neighboring artery was demonstrated by subtraction CTA in 1 patient and by conventional CTA in 3 (Fig 3).

\section{Discussion}

Our study showed that OSHST was a useful technique for eliminating the artifact generated by cobalt-alloy clips and was available for evaluating status following surgical placement of clips.

To get the subtraction images, we obtained 2 consecutive scans and subtracted the first scan from second scan. For subtraction CT with OSHST, an initial scan was obtained followed by a second scan with the initial tube angle synchronized to the first scan, so that an essentially identical helical path was scanned. For subtraction CT without OSHST, the initial tube angle of 2 consecutive helical scans was randomly chosen so that a different helical path was scanned. Because helical CT scans along a helical orbit, different helical orbits make the misregistration, even if images are acquired in the same position. When we evaluated surgically clipped aneurysms, streak artifacts induced by clips appeared in the same directions in 2 scans with OSHST; subtraction images eliminated potential clip artifacts (Fig 1).

Several reports ${ }^{5-9}$ have discussed the postclipping evaluation of cerebral aneurysms by using CTA, most in cases using titanium-alloy clips. The quality of conventional CTA was reported as acceptable in $42 / 49(86 \%)$ patients with titaniumalloy clips. ${ }^{5}$ We reported that subtraction CTA with OSHST showed applicable image quality at the cobalt-alloy clip site in $95 \%$ of postoperative aneurysms, excellent findings compared 

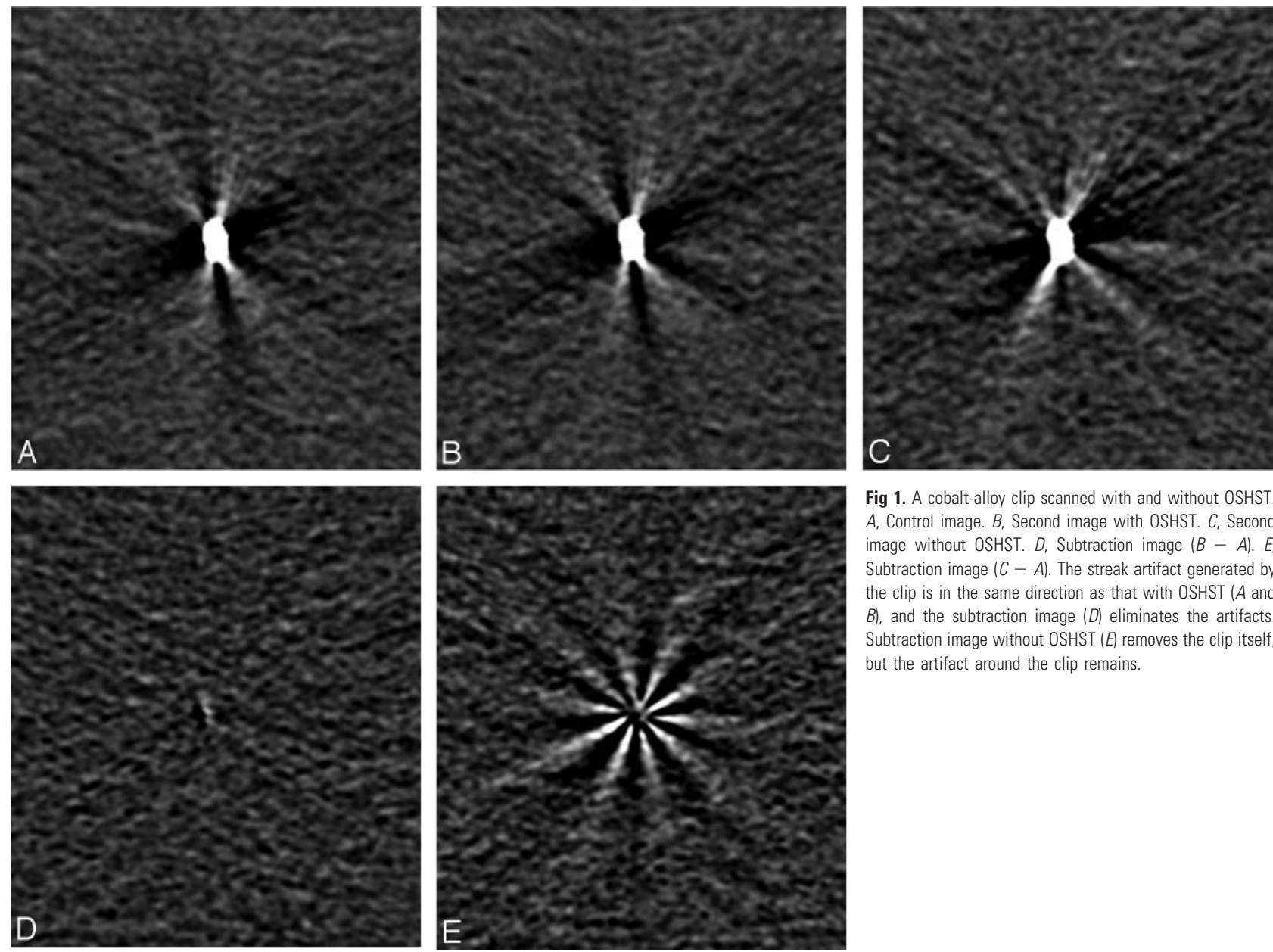

Fig 1. A cobalt-alloy clip scanned with and without OSHST $A$, Control image. B, Second image with OSHST. C, Second image without OSHST. D, Subtraction image $(B-A)$. $E$ Subtraction image $(C-A)$. The streak artifact generated by the clip is in the same direction as that with OSHST I $A$ and $B)$, and the subtraction image $(D)$ eliminates the artifacts. Subtraction image without OSHST (E) removes the clip itself, but the artifact around the clip remains.

with those from previous studies. Sagara et $\mathrm{al}^{8}$ showed that cobalt-alloy clips generate strong metal artifacts that interfere with evaluation in conventional CTA studies. van der Schaaf et $\mathrm{al}^{9}$ reported that CTA could not evaluate the clip site in $62 \%$ of patients treated with cobalt-alloy clips. They used the subtraction method with matched bone elimination, ${ }^{3}$ which makes the bone mask image by noncontrast imaging. Although bone mask subtraction might delete the clips themselves, the artifacts generated by the clips were difficult to remove by using this method.

It is reported that residual necks remain after aneurysm clipping in approximately $5 \%$ of patients and that aneurysms re-expand in $2.9 \%$ of patients. ${ }^{10,11}$ Long-term follow-up has also revealed new aneurysms in $8 \%$ of patients after subarachnoid hemorrhage, ${ }^{12,13}$ indicating the importance of postoperative follow-up. To date, postoperative status has usually been evaluated by using DSA. Postclipping evaluation with conventional CTA depended on the alloy materials of the clips and was considered viable only with clips made from titanium alloy. Here, we show that subtraction CTA with OSHST allows evaluation after operative placement of cobalt-alloy clips.

To reduce the clip-induced artifact, van der Schaaf et al ${ }^{14}$ suggested scanning with high kilovoltage $(140 \mathrm{kV})$, low helical pitch (0.6), and high-concentration contrast medium (370 mg $\mathrm{I} / \mathrm{mL}$ ). Clip angle to scanning plane also influenced the size of artifact, so a clip positioned perpendicular to the scanning plane led to fewer artifacts. ${ }^{8,14}$ Adjusting the head angle was

\begin{tabular}{llll}
\hline \multicolumn{2}{l}{ Table 1: Summary of clip phantom study* } \\
\hline & \multicolumn{2}{c}{ CT Value } & \multirow{2}{*}{ X-ray Tube } \\
OSHST & Average & SD & Angle \\
\hline Subtraction with & $0.1 \pm 0.0$ & $14.2 \pm 1.3 \dagger$ & $0.9 \pm 0.5 \dagger$ \\
Subtraction without & $0.1 \pm 0.1$ & $23.1 \pm 1.9$ & $127.3 \pm 86.3$ \\
\hline
\end{tabular}

* Data are expressed by mean $\pm \mathrm{SD}$.

$\dagger P<.05$ compared with subtraction without OSHST.

limited for patients' examinations, and an angled clip or multiple clips are difficult to adjust in the scanning plane. Our subtraction method was less affected by clip angles and numbers. However for image subtraction, small clip-induced artifacts were preferred to large artifacts, and a specific exposure dose was required.

Because subtraction CTA with OSHST requires a noncontrast scan that must be obtained under identical scanning conditions, this technique requires double exposure to radiation. Bone-subtraction CTA with matched bone elimination allows a noncontrast image acquisition with the radiation dose reduced by one third to one fourth. ${ }^{2,3,15}$ If we reduced the radiation dose for non-contrast scans with subtraction CTA with OSHST, the extent of artifact generated by clips were different, and complete subtraction of artifacts might be impossible. The overall exposure dose could be reduced if DSA was avoided.

The disadvantage of subtraction CTA with OSHST is that a head-immobilization device is required, and patient preparation for CTA is rather complicated and time-consuming. We 


\begin{tabular}{|c|c|c|c|c|c|c|c|}
\hline \multirow[b]{2}{*}{ DSA Findings } & \multicolumn{3}{|c|}{ Subtraction CTA } & \multicolumn{3}{|c|}{ Conventional CTA } & \multirow[b]{2}{*}{ Total } \\
\hline & Positive & Negative & NA & Positive & Negative & NA & \\
\hline \multicolumn{8}{|l|}{ Residual neck } \\
\hline Positive & 4 & 0 & 0 & 1 & 0 & 3 & 4 \\
\hline Negative & 0 & 15 & 1 & 0 & 4 & 12 & 16 \\
\hline \multicolumn{8}{|c|}{$\begin{array}{l}\text { Parent or neighboring artery steno-occlusive } \\
\text { change }\end{array}$} \\
\hline Positive & 1 & 0 & 0 & 0 & 0 & 1 & 1 \\
\hline Negative & 1 & 18 & 0 & 3 & 6 & 10 & 19 \\
\hline
\end{tabular}

Note:-NA indicates not applicable for diagnosis due to clip-induced artifact or inappropriate subtraction.
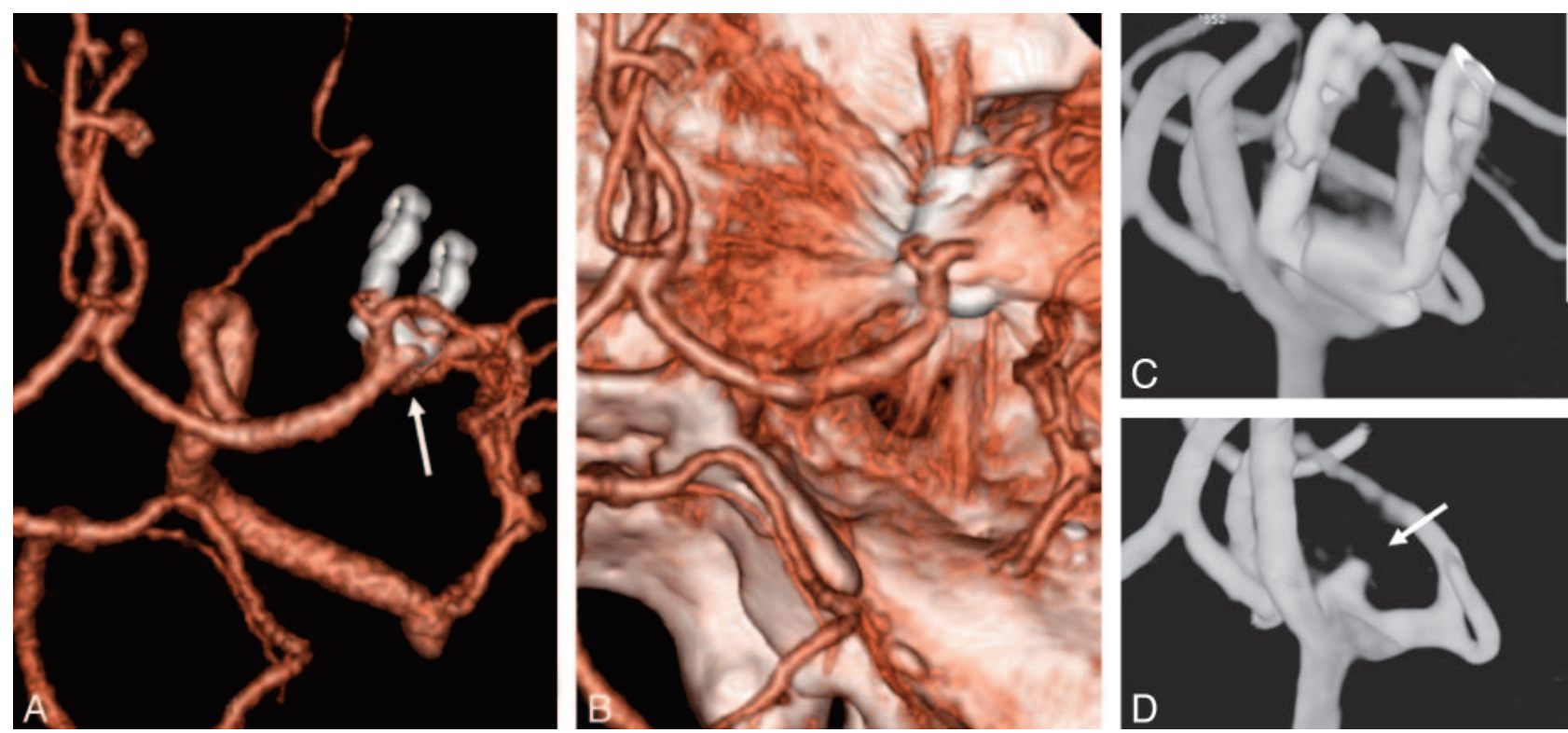

Fig 2. One-year follow-up of a 46-year-old man who underwent clipping of an aneurysm of the right middle cerebral artery with 2 cobalt-alloy clips. $A$, Subtraction CTA. B, Conventional CTA. C, 3D DSA with clips. D, 3D DSA without clips. Subtraction CTA (A) and 3D DSA (C and D) represent re-expansion of the aneurysm (arrows). Visualizing the aneurysm on conventional CTA $(B)$ is complicated by a large artifact arising from the clips.
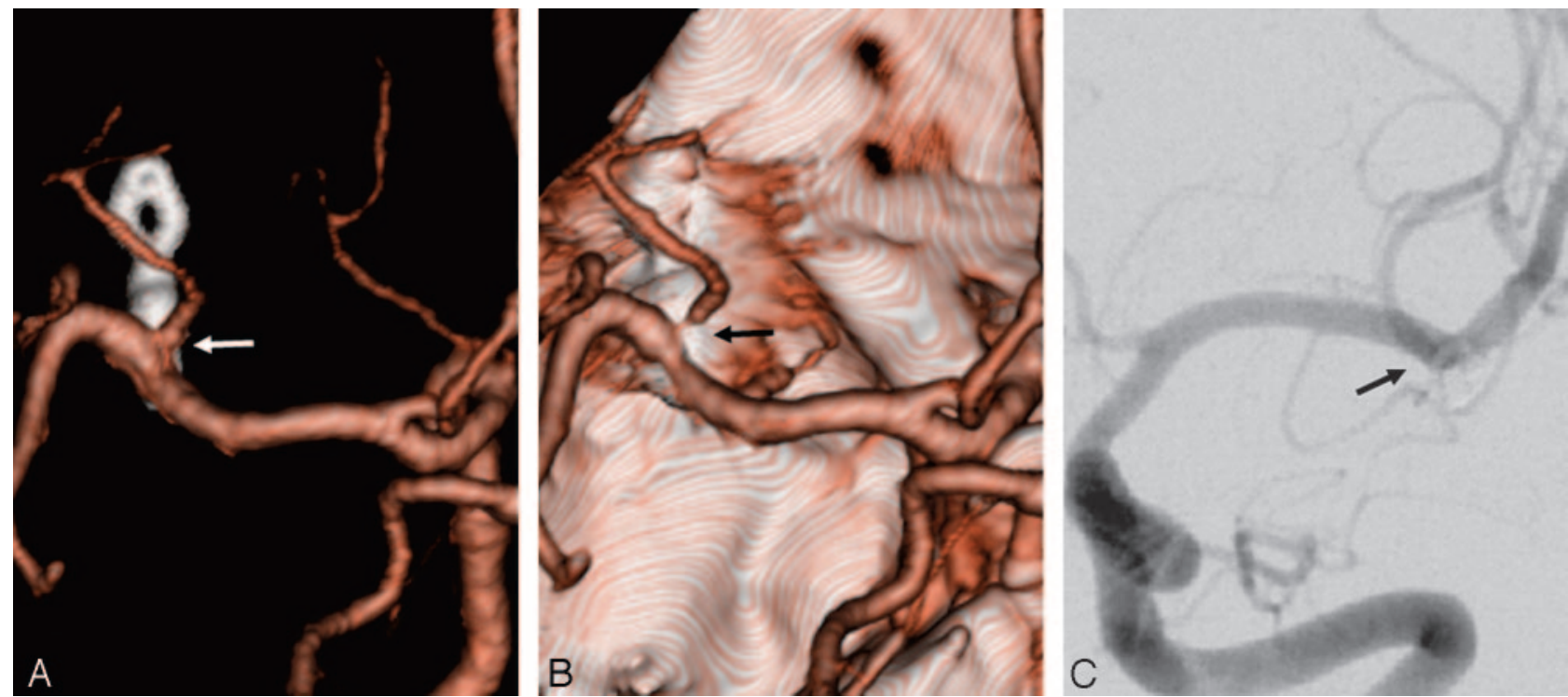

Fig 3. Two-month follow-up of a 38-year-old man who underwent clipping of a left middle cerebral artery aneurysm with a cobalt-alloy clip. $A$, Subtraction CTA. $B$, Conventional CTA. $C$, DSA of the left internal carotid artery. Conventional CTA (B) represents the pseudostenosis (arrow) at proximal M2 near the clipping site.

used subtraction CTA with OSHST to evaluate aneurysms surgically treated with cobalt-alloy clips. In particular, $75 \%$ (15/ 20) of patients were difficult to evaluate by using conventional
CTA, compared with 5\% (1/20) who could not undergo neck evaluation by using subtraction CTA with OSHST. Subtraction CTA with OSHST allows replacement of DSA with less- 
invasive and cheaper CTA in patients with aneurysms surgically treated with cobalt-alloy clips, minimizes patient discomfort, and should reduce medical costs.

\section{Conclusions}

In this study, subtraction CTA with OSHST is an accurate noninvasive tool for assessment of postoperative aneurysms with cobalt-alloy clips and could be used as an alternative to DSA.

\section{References}

1. Sakamoto S, Kiura Y, Shibukawa M, et al. Subtracted 3D CT angiography for evaluation of internal carotid artery aneurysms: comparison with conventional digital subtraction angiography. AJNR Am J Neuroradiol 2006;27:1332-37

2. Tomandl BF, Hammen T, Klotz E, et al. Bone-subtraction CT angiography for the evaluation of intracranial aneurysms. AJNR Am J Neuroradiol 2006;27:55-59

3. Venema HW, Hulsmans FJ, den Heeten GJ. CT angiography of the circle of Willis and intracranial internal carotid arteries: maximum intensity projection with matched mask bone elimination-feasibility study. Radiology 2001;218:893-98

4. Imakita S, Onishi Y, Hashimoto T, et al. Subtraction CT angiography with controlled-orbit helical scanning for detection of intracranial aneurysms. AJNR Am J Neuroradiol 1998;19:291-95

5. Dehdashti AR, Binaghi S, Uske A, et al. Comparison of multislice computerized tomography angiography and digital subtraction angiography in the postoperative evaluation of patients with clipped aneurysms. J Neurosurg 2006;104:395-403

6. Sakuma I, Tomura N, Kinouchi H, et al. Postoperative three-dimensional CT angiography after cerebral aneurysm clipping with titanium clips: detection with single detector CT-comparison with intra-arterial digital subtraction angiography. Clin Radiol 2006;61:505-12

7. Teksam M, McKinney A, Cakir B, et al. Multi-slice computed tomography angiography in the detection of residual or recurrent cerebral aneurysms after surgical clipping. Acta Radiol 2004;45:571-76

8. Sagara Y, Kiyosue H, Hori Y, et al. Limitations of three-dimensional reconstructed computerized tomography angiography after clip placement for intracranial aneurysms. J Neurosurg 2005;103:656-61

9. van der Schaaf IC, Velthuis BK, Wermer MJ, et al. Multislice computed tomography angiography screening for new aneurysms in patients with previously clip-treated intracranial aneurysms: feasibility, positive predictive value, and interobserver agreement. J Neurosurg 2006;105:682-88

10. Thornton J, Bashir Q, Aletich VA, et al. What percentage of surgically clipped intracranial aneurysms have residual necks? Neurosurgery 2000;46:1294-98, discussion 1298-1300

11. Tsutsumi K, Ueki K, Morita A, et al. Risk of aneurysm recurrence in patients with clipped cerebral aneurysms: results of long-term follow-up angiography-what percentage of surgically clipped intracranial aneurysms have residual necks? Stroke 2001;32:1191-94

12. Wermer MJ, Buskens E, van der Schaaf IC, et al. Yield of screening for new aneurysms after treatment for subarachnoid hemorrhage. Neurology 2004;62:369-75

13. Wermer MJ, van der Schaaf IC, Velthuis BK, et al. Follow-up screening after subarachnoid haemorrhage: frequency and determinants of new aneurysms and enlargement of existing aneurysms. Brain 2005;128:2421-29

14. van der Schaaf I, van Leeuwen M, Vlassenbroek A, et al. Minimizing clip artifacts in multi CT angiography of clipped patients. AJNR Am J Neuroradiol 2006;27:60-66

15. Venema HW, den Heeten GJ. Subtraction helical CT angiography of intra- and extracranial vessels: technical considerations and preliminary experiencerediscovery of matched mask bone elimination? AJNR Am J Neuroradiol 2003 24:1491, author reply 1491-14 